\title{
Disturbance Feedforward Control for Vibration Suppression in Adaptive Optics of Large Telescopes
}

\author{
Martin Glück ${ }^{\mathrm{a}, \mathrm{b}}$, Jörg-Uwe Pott ${ }^{\mathrm{b}}$, and Oliver Sawodny ${ }^{\mathrm{a}}$ \\ ${ }^{a}$ Institute for System Dynamics, University of Stuttgart, Waldburgstr. 17/19, 70563 Stuttgart, \\ Germany \\ bMax Planck Institute for Astronomy, Königsstuhl 17 , 69117 Heidelberg, Germany
}

\begin{abstract}
With increasing the telescope size the effects of structural vibrations become more dominant and therefore its suppression in Adaptive Optics system is a big challenge. For that reason new control concepts as the observerbased disturbance compensation (LQG control) were developed. Nevertheless, under different environmental conditions these concepts are not sufficient to suppress the vibrations in an acceptable range. When observing with faint natural guide stars the integration time of the wavefront sensor is increased to obtain a better Signalto-Noise ratio. However by reducing the loop speed the bandwidth of the AO system cannot mitigate high frequency vibrations $(>5 \mathrm{~Hz}$ ). In this paper an additional accelerometer-based disturbance feedforward (DFF) control is proposed to improve the performance for high frequency vibrations. In an AO end-to-end simulation the DFF control is compared with the common concepts. Thereby, it is shown that the Strehl ratio can be increased for faint natural guide stars $(>13 \mathrm{mag})$ by a factor two up to four.
\end{abstract}

Keywords: Adaptive Optics, Telescope Vibrations, Disturbance Compensation, Disturbance Observer, Disturbance Feedforward Control

\section{INTRODUCTION}

Reaching the diffraction limit of large telescopes is mainly disturbed by atmospheric turbulences and structural vibrations. ${ }^{1,2}$ Nowadays, these disturbances are compensated by Adaptive Optics (AO) systems. However, in the last decades the construction of larger telescopes generates more dominant vibrations at the telescope structure. Therefore, the vibrations at the telescope mirrors deserve special attention. Because of the mirror mounting high frequency vibrations especially in the tip-tilt modes are induced into the optical path. These vibrations cannot be mitigated efficiently by a classical integral control system and thus new concepts such as the Linear Quadratic Gaussian control were developed. ${ }^{3}$ But in particular for the observation with faint Natural Guide Stars (NGS) there is a fundamental limitation for the suppression of high frequency vibrations. To achieve a sufficient Signal-To-Noise-Ratio the exposure time of the wavefront sensor is increased. Therefore, the bandwidth of the control system is decreased and thus the high frequency vibrations worsen the optical performance. To suppress these vibrations we developed a disturbance feedforward (DFF) control concept based on an additional fast vibration measurement system. At the Large Binocular Telescope (LBT) there are already exits accelerometers which can be used for investigating the concept. On each mirror (primary, secondary and tertiary mirror) are mounted several accelerometers to determine the low order aberrations (Piston, Tip, Tilt). As accelerometers unidirectional piezoelectric sensors are used which are sampled with $1 \mathrm{kHz}$. Therefore, frequencies in a range between $5 \mathrm{~Hz}$ and $500 \mathrm{~Hz}$ can be detected. Based on the accelerometer data the piston, tip and tilt modes are reconstructed with suitable algorithms ${ }^{4,5}$ and the control signal for the adaptive mirrors of the AO system are determined. Suppressing the piston mode is in regular use on sky in the LBT Interferometer (LBTI). ${ }^{6}$ For the suppression of the tip-tilt modes we simulated the First Light AO (FLAO) system with an accelerometer-based disturbance feedforward (DFF) control system and we achieved an improvement of the Strehl ratio by an factor of 2 to $4 .^{7}$ The disturbance feedforward control cannot only be used for Single Conjugated AO (SCAO) systems but also for Multi Conjugated AO (MCAO) systems. The tip-tilt NGS can be focused on fainter guide stars and hence, the sky-coverage can be increased.

Further author information: E-mail: martin.glueck@isys.uni-stuttgart.de 
In this paper the common controls concepts for compensating vibrations in the AO system of large telescocpes are presented in section 2. Afterwards the accelerometer-based disturbance feedforward (DFF) control is explained more in detail. In section 4 the introduced concepts are compared and the benefit of a DFF control is shown.

\section{COMMON VIBRATION SUPPRESSION CONCEPTS}

In Adaptive Optics there are several concepts to suppress vibration of the telescope structure. In this section two common concepts, an integral control and a obersever-based compensation method, are presented and later compared with our proposed disturbance feedforward control (DFF). The typical AO structure is represented in the following Fig. 1. The optical light pass through the telescope structure is disturbed by the atmosphere

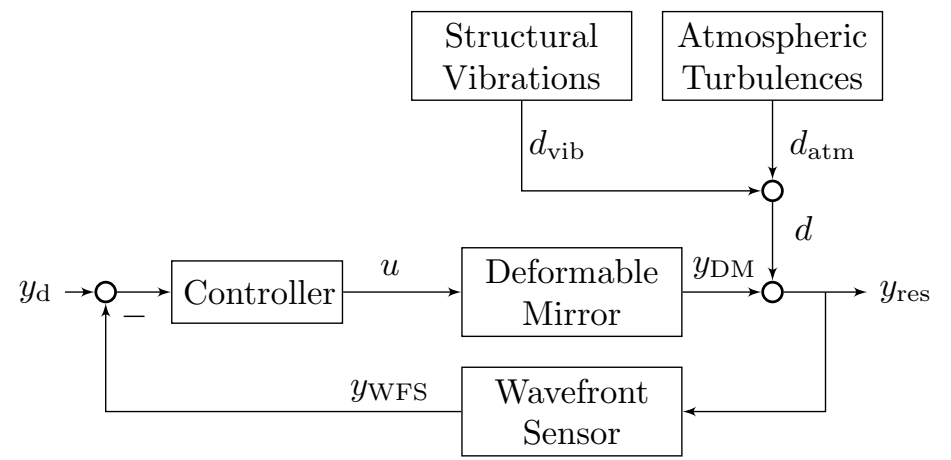

Figure 1. Overview of the control structure of the typical Adaptive Optics system in large telescopes

$d_{\text {atm }}$ and the structural vibrations $d_{\text {vib }}$. Within the path a deformable mirror (DM) is positioned to compensate the mentioned disturbances. To determine the control signal of the DM $u$ the residual wavefront error $y_{\text {res }}$ is measured by a wavefront sensor e.g. Shack-Hartmann or Pyramid sensor. Typically, the measurement signal $y_{\text {WFS }}$ is delayed by 2 two time samples, one for the exposure and one for the wavefront reconstruction. In control theory this structure is also known as a disturbance compensation, because the control goal $y_{\mathrm{d}}$ is to bring the residual output to zero. To reach a disturbance rejection the most used concept is an integral control. This controller feeds back the integral of the measured residual wavefront error

$$
u[k]=u[k-1]-g y_{\mathrm{WFS}}[k] .
$$

$g$ is the loop gain. In comparison to the integral control approach a disturbance observer-based compensation is used. Here, we want to mention that the name observer is to understand in the context of control theory. Observers are used to estimate the state of a dynamic system by using the measurement and input signals. In the AO community it is often used the LQG control,e.g. Petit et al. ${ }^{8}$ who uses the Kalman Filter for the state estimation. The idea is to design a disturbance observer based on a disturbance model. A dynamic of the atmosphere can be derived from the statistical description of Kolmogorov, see Hardy. ${ }^{9}$ The spatial power spectral density (PSD) is given in the following

$$
\Phi\left(f_{\mathrm{x}}, f_{\mathrm{y}}\right)=0.023 r_{0}^{-\frac{5}{3}}\left(f_{\mathrm{x}}^{2}+f_{\mathrm{y}}^{2}\right)^{-\frac{11}{6}}
$$

$f_{x} f_{y}$ are spatial frequencies and the $r_{0}$ is the Fried parameter which is an expression for the seeing. ${ }^{9}$ To get a connection to the temporal behavior of the atmosphere it is used the Taylor Hypothesis, which assumes a frozen wavefront with a constant velocity $v .^{9}$ This assumption is justified for small sample times. In AO system the sample times are in a range between $100 \mathrm{~Hz}$ and $1 \mathrm{kHz}$, which allows the use of the Taylor Hypothesis. Using this assumption a mathematical connection of the temporal and the spatial autocorrelation exits ${ }^{10}$

$$
R(\tau)=R_{\mathrm{S}}\left(v_{x} \tau, v_{y} \tau\right)
$$


$\tau$ is the temporal and $\tau_{x}, \tau_{y}$ the spatial shift parameter. The spatial autocorrelation can now be calculated from the power spectral density with the Wiener-Khinchin theorem

$$
R_{\mathrm{S}}\left(\tau_{x}, \tau_{y}\right)=\int_{-\infty}^{\infty} \int_{-\infty}^{\infty} \Phi\left(f_{\mathrm{x}}, f_{\mathrm{y}}\right) e^{j 2 \pi\left(f_{\mathrm{x}} \tau_{x}+f_{\mathrm{y}} \tau_{y}\right)} d f_{\mathrm{x}} d f_{\mathrm{y}}
$$

Hence, the temporal PSD can now be determined from the spatial PSD

$$
\Phi_{\mathrm{T}}(f)=\int_{-\infty}^{\infty} R_{\mathrm{S}}\left(v_{x} \tau, v_{y} \tau\right) e^{-j 2 \pi f \tau} d \tau .
$$

$f$ is the temporal frequency. To derive a dynamic model the temporal PSD is approximated by an second order Autoregressive (AR) model

$$
\Phi_{\mathrm{T}}(f)=\frac{\sigma^{2}}{\left|1-\sum_{m=1}^{2} a_{m} e^{-j 2 \pi f m}\right|^{2}}
$$

$\sigma^{2}$ is the variance of the atmosphere signal and $a_{m}$ the coefficients of the AR model. Based on this equation a state space representation is determined, see Meimon ${ }^{11}$

$$
\begin{aligned}
& \underbrace{\left[\begin{array}{c}
d_{\mathrm{atm}, i}[k] \\
d_{\mathrm{atm}, i}[k-1]
\end{array}\right]}_{x_{\mathrm{atm}, i}[k+1]}=\underbrace{\left[\begin{array}{cc}
a_{1} & a_{2} \\
1 & 0
\end{array}\right]}_{A_{\mathrm{atm}, i}} \underbrace{\left[\begin{array}{c}
d_{\mathrm{atm}, i}[k-1] \\
d_{\mathrm{atm}, i}[k-2]
\end{array}\right]}_{x_{\mathrm{atm}, i}[k]}+\left[\begin{array}{l}
1 \\
0
\end{array}\right] v_{\mathrm{atm}}[k] \\
& d_{\mathrm{atm}}[k-1]=\underbrace{\left[\begin{array}{cc}
1 & 0
\end{array}\right]}_{C_{\mathrm{atm}}} x_{\mathrm{atm}}[k]
\end{aligned}
$$

$v_{\text {atm }}$ is a random input. The vibrations are modeled by a discrete mechanical modal model ${ }^{12}$

$$
\begin{aligned}
& \underbrace{\left[\begin{array}{c}
d_{\mathrm{vib}, i}[k] \\
d_{\mathrm{vib}, i}[k-1]
\end{array}\right]}_{x_{\mathrm{vib}, i}[k+1]}=\underbrace{\left[\begin{array}{cc}
2 e^{-\omega_{0, i} \delta_{i} T_{\mathrm{s}}} \cos \left(\omega_{0, i} T_{\mathrm{s}} \sqrt{1-\delta_{i}^{2}}\right) & -e^{-2 \omega_{0, i} \delta_{i} T_{\mathrm{s}}} \\
1
\end{array}\right]}_{A_{\mathrm{vib}, i}} \underbrace{\left[\begin{array}{c}
d_{\mathrm{vib}, i}[k-1] \\
d_{\mathrm{vib}, i}[k-2]
\end{array}\right]}_{x_{\mathrm{vib}, i}[k]}+\left[\begin{array}{l}
1 \\
0
\end{array}\right] v_{\mathrm{vib}[k]} \\
& d_{\mathrm{vib}}[k-1]=\underbrace{\left[\begin{array}{ll}
1 & 0
\end{array}\right]}_{C_{\mathrm{vib}}} x_{\mathrm{vib}}[k]
\end{aligned}
$$

The natural frequencies $\omega_{0, i}$ and the damping coefficients $\delta_{i}$ can be measured at the telescope structure. $v_{\mathrm{vib}}$ is also a random input. Both model can be combined to a entire disturbance model

$$
d[k]=d_{\mathrm{atm}}[k]+d_{\mathrm{vib}}[k] .
$$

The disturbances are estimated by using the wavefront sensor measurements, which measures the residual error

$$
y_{\mathrm{WFS}[k]}=d[k-1]-u[k-2] .
$$

$d[k-1]$ is the mean over the disturbed wavefront from $k-2$ to $k-1$ and $u[k-2]$ the control signal of the DM. Therefore, the control signal has to be set one time step before the sensor integration. Here, the dynamic of the DM is neglected because of its fast dynamics. The delays in the disturbances can be considered in the AR model. But the input delay is described by a classical discrete state space description

$$
\begin{aligned}
x_{\mathrm{T}}[k+1] & =\underbrace{\left[\begin{array}{ll}
1 & 0 \\
0 & 0
\end{array}\right]}_{A_{\mathrm{T}}} x_{\mathrm{T}}[k]+\underbrace{\left[\begin{array}{l}
1 \\
0
\end{array}\right]}_{B_{\mathrm{T}}} u[k] \\
u[k-2] & =\underbrace{\left[\begin{array}{ll}
0 & 1
\end{array}\right]}_{C_{\mathrm{T}}} x_{\mathrm{T}}[k] .
\end{aligned}
$$


Based on the introduced models a state space representation of the whole system can be determined

$$
\begin{aligned}
& x[k+1]=\underbrace{\left[\begin{array}{ccc}
A_{\mathrm{atm}} & 0 & 0 \\
0 & A_{\mathrm{vib}} & 0 \\
0 & 0 & A_{T}
\end{array}\right]}_{A} \underbrace{\left[\begin{array}{c}
x_{\mathrm{atm}}[k] \\
x_{\mathrm{vib}}[k] \\
x_{\mathrm{T}}[k]
\end{array}\right]}_{x}+\underbrace{\left[\begin{array}{c}
0 \\
0 \\
B_{\mathrm{T}}
\end{array}\right]}_{B} u[k]+\left[\begin{array}{c}
v_{\mathrm{atm}} \\
v_{\mathrm{vib}} \\
0
\end{array}\right] \\
& y_{\mathrm{WFS}}[k]=\underbrace{\left[\begin{array}{lll}
C_{\mathrm{atm}} & C_{\mathrm{vib}} & -C_{\mathrm{T}}
\end{array}\right]}_{C} x[k]
\end{aligned}
$$

Now, the disturbance observer can be designed. For Linear System it is often used a Luenberger Observer or a Kalman Filter. In the astronomy AO community the Kalman Filter is commonly used. The Kalman Filter estimates the states by minimizing the mean square error of the state. The Kalman Filter is described with the following equations ${ }^{13}$

$$
\begin{aligned}
\text { Prediction: } & \\
\hat{x}[k \mid k-1] & =A \hat{x}[k-1]+B u[k-1] \\
P[k \mid k-1] & =A P[k-1] A^{T}+Q
\end{aligned}
$$

Correction:

$$
\begin{aligned}
L[k] & =P[k \mid k-1]=C^{T}\left(C P[k \mid k-1] C^{T}+R\right)^{-1} \\
\hat{x}[k] & =\hat{x}[k \mid k-1]+L[k]\left(y_{\mathrm{WFS}}[k]-C \hat{x}[k \mid k-1]\right) \\
P[k] & =(I-L[k] C) P[k \mid k-1] .
\end{aligned}
$$

$Q$ is the process noise and $R$ the sensor noise. Based on the estimated state we can feed the DM with the negative disturbance signal

$$
u[k]=-\left[\begin{array}{llllll}
1 & 0 & 1 & 0 & 0 & 0
\end{array}\right] \hat{x}[k] .
$$

\section{DISTURBANCE FEEDFORWARD CONTROL}

The concept of the DFF control is nearby similar to the obserber-based disturbance compensation. The difference is that the vibrational disturbances are directly measured by the accelerometers at the telescope structure. Based on the accelerometer data the disturbances in the optical modes are reconstructed and directly given to the input of the DM. One advantage is that there is no feedback and therefore the AO system cannot be unstable. Moreover, the system can be operated with different sample rates in comparison to the feedback loop. Hence, the DFF can increase the bandwidth of the AO system for the observation with faint natural guide stars. For compensation the atmosphere and the residual error of the DFF a classical integral control is used. The DFF structure is depicted in Fig. 3.

An online reconstruction of the tip-tilt cannot be done by a classical double integration, because it is unstable. For that reason, new reconstruction methods were developed. A typical method is to estimate the tip-tilt by a Kalman Filter or as known from section 2 by a Luenberber observer. ${ }^{4,14}$ Böhm et al. ${ }^{15}$ proposes a bandpass filter. The benefit is that you don't need a dynamical model of the telescope structure. Vibrations can be suppressed in a large frequency range $(5 \mathrm{~Hz}-50 \mathrm{~Hz})$. But the suppression is not optimal for the whole range because of the phase characteristics of the bandpass filter. ${ }^{16}$ Another typical problem in such large system is the time delay between accelerometer and the DM. By using the Kalman filter as disturbance observer, the time delay can be considered within the disturbance model. An alternative time delay approach from Böhm ${ }^{17}$ can be used without a model. Moreover, for the disturbance feedforward it is important that the dynamics of the DM can be neglected. Otherwise the inverse dynamics has to considered on the input signal at the DM.

\section{SIMULATION RESULTS}

The three presented vibration disturbance compensation methods are simulated in an AO end-to-end simulation for different magnitudes of the guide star. At first, an ideal case, bright guide star with 10.5 mag in R-Band, 


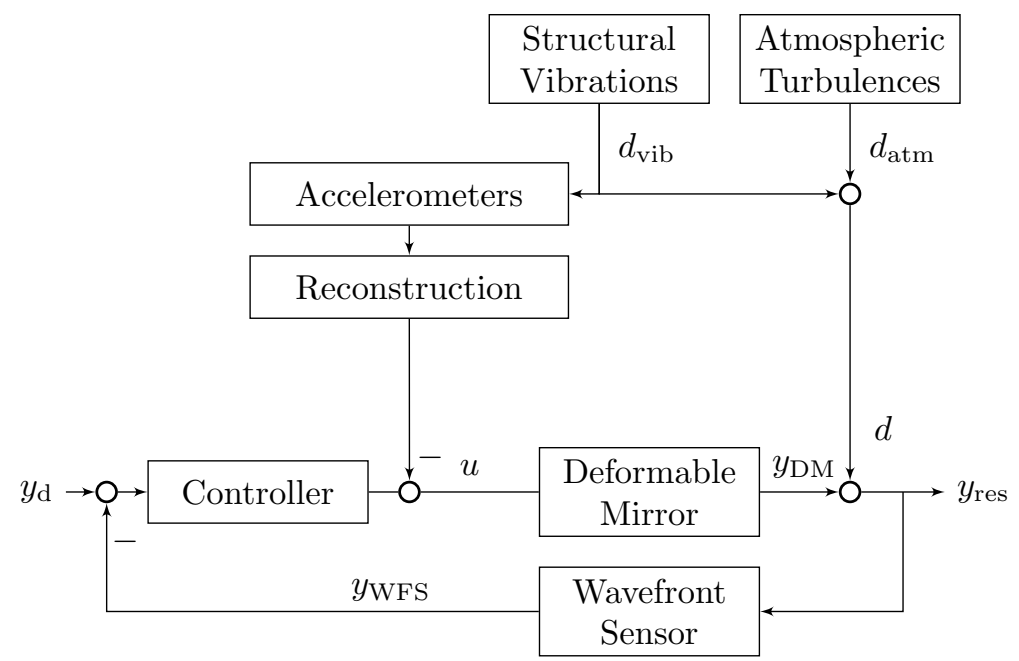

Figure 2. Overview of the control structure of the Adaptive Optics system with an additional disturbance feedforward control

$800 \mathrm{~Hz}$ sample rate and 0.8 arcsec seeing, is depicted in Fig. 3. The system is excited by an single natural frequency that is swept from $1 \mathrm{~Hz}$ to $50 \mathrm{~Hz}$ and the amplitude is changed from 25 mas to 150 mas. It is shown that the performance of a classical integral control is only useful for small amplitudes and frequencies up to $10 \mathrm{~Hz}$. In comparison to that the disturbance observer-based compensation increases the frequency range up to $20 \mathrm{~Hz}$ also for large amplitudes. The DFF delivers a good compensation for the whole frequency range. Because the DFF isn't in the feedback path, a much better vibration suppression can be achieved. Of course effects such as sensor noise, reconstruction errors and time delays worsens the performance of the DFF, but in this case the effects are small. In comparison to bright guide star we consider a faint natural guide star 14.6 mag with

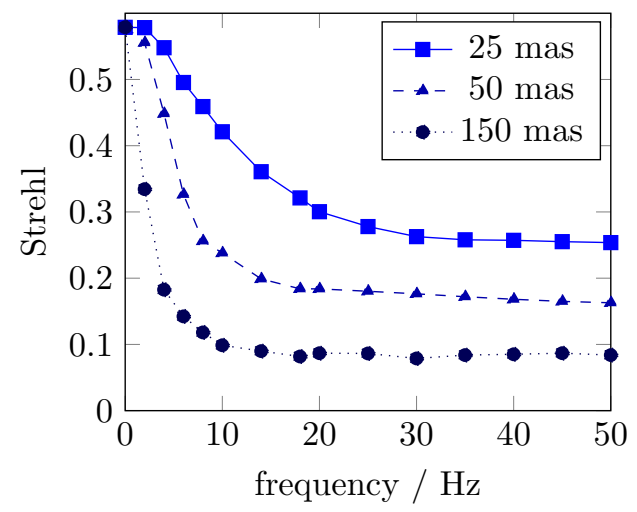

(a) Integral

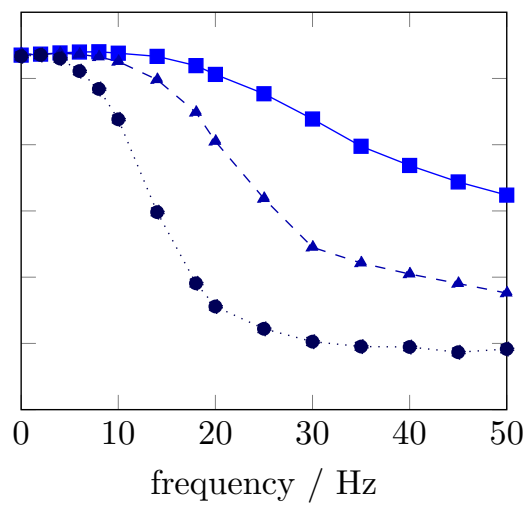

(b) LQG

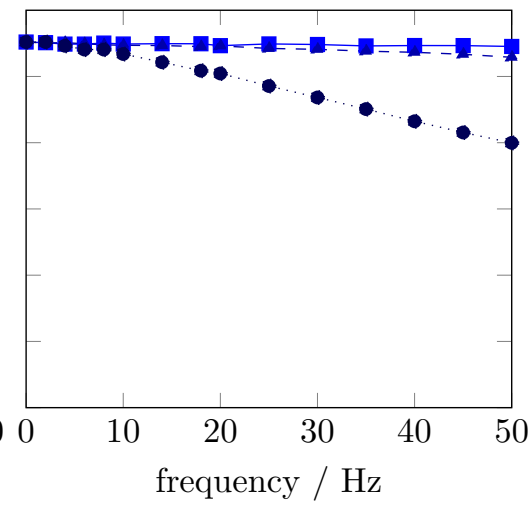

(c) $\mathrm{DFF}$

Figure 3. Simulation results for a observation with a bright natural guides star 10.5 mag,seeing 0.8 arcsec and sample rate $800 \mathrm{~Hz}$. The AO system is excited with a single sine function by changing the amplitude and frequency. The disturbance is compensated by the three presented disturbance compensation concepts.

0.8 arcsec seeing, $200 \mathrm{~Hz}$ sample rate, see Fig 4. As we expect the strehl decreases because it is used much less optical modes for the compensation. In this scenario the effect of the slow sample time is also seen in the Strehl of the observer-based compensation. The strehl is decreased by a factor 2 at $20 \mathrm{~Hz}$ with only 25 mas excitation amplitude. In this case the benefit of a DFF is clearly visible and is recommended for the observation with faint natural guide stars. 


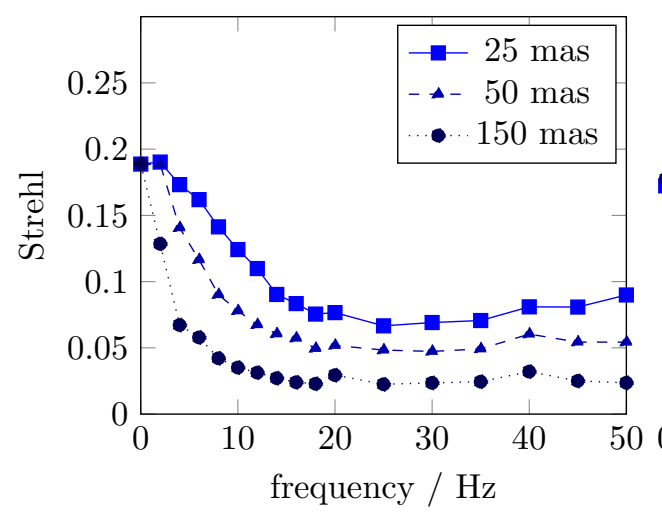

(a) Integral

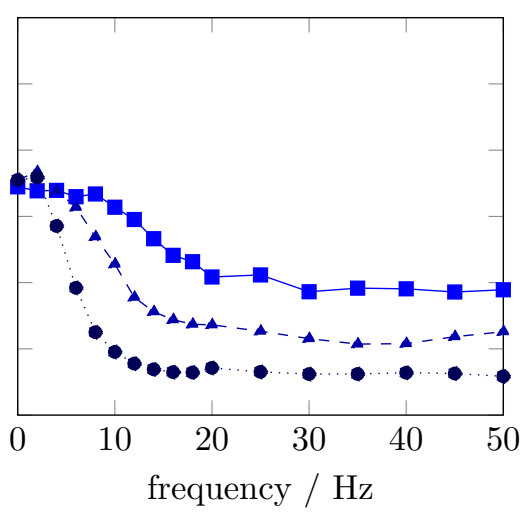

(b) LQG

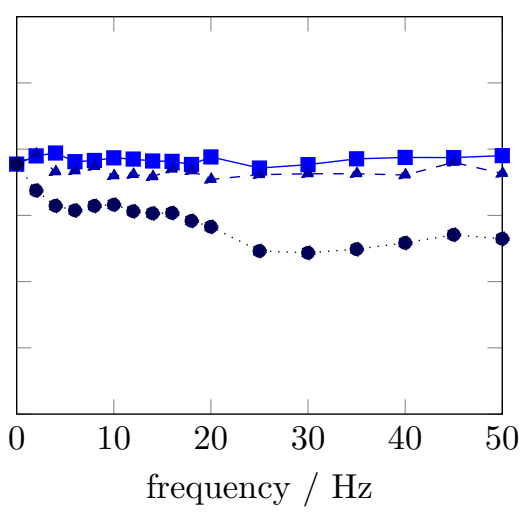

(c) DFF

Figure 4. Simulation results for a faint natural guide star $14.6 \mathrm{mag}$, seeing 0.8 arcsec and sample rate $200 \mathrm{~Hz}$.

\section{CONCLUSION}

In this paper investigations on the suppression of vibrations in Adaptive Optics of large telescopes has been presented. We have proposed an additional accelerometer-based disturbance feedforward control and have compared it with the two common suppression concepts the integral and LQG control. Thereby, it is shown that the DFF especially improves the AO system for high frequency vibrations $(>5 \mathrm{~Hz})$ and faint natural guide star sources $(>13 \mathrm{mag})$. For that special scenario the Strehl Ratio can be increased by a factor two to four. The proposed concept is an easy and cheap solution to increase the performance range of an instrument in large telescope in particular for $\mathrm{AO}$ conditions of windy nights. Moreover, it could be a method to handle the foreseen large vibrational influences in extremely large telescopes as the ELT.

\section{REFERENCES}

[1] Clenet, Y., Kasper, M. E., Ageorges, N., Lidman, C., Fusco, T., Marco, O., Hartung, M., Mouillet, D., Koehler, B., Rousset, G., and et al., "Naco performance: status after 2 years of operation," Advancements in Adaptive Optics (Oct 2004).

[2] Brix, M., Naranjo, V., Beckmann, U., Bertram, R., Bertram, T., Brynnel, J., Egner, S., Gaessler, W., Herbst, T. M., Kuerster, M., Rohloff, R. R., Rost, S., and Schmidt, J., "Vibration measurements at the large binocular telescope (lbt)," (2008).

[3] Petit, C., Conan, J.-M., Kulcsr, C., Raynaud, H.-F., and Fusco, T., "First laboratory validation of vibration filtering with lqg control law for adaptive optics," Optics Express 16(1), 87 (2008).

[4] Böhm, M., Pott, J.-U., Sawodny, O., Herbst, T., and Kürster, M., "Real-time vibration compensation for large telescopes," Monthly Notices of the Royal Astronomical Society 442(3), 2446-2455 (2014).

[5] Keck, A., Pott, J.-U., and Sawodny, O., "Accelerometer-based online reconstruction of vibrations from delayed measurements," 2015 IEEE Conference on Control Applications (CCA) (Sep 2015).

[6] Böhm, M., Pott, J.-U., Borelli, J., Hinz, P., Defrère, D., Downey, E., Hill, J., Summers, K., Conrad, A., Kürster, M., and et al., "Ovms-plus at the lbt: disturbance compensation simplified," Ground-based and Airborne Telescopes VI (Jul 2016).

[7] Glück, M., Pott, J.-U., and Sawodny, O., "Investigations of an accelerometer-based disturbance feedforward control for vibration suppression in adaptive optics of large telescopes," Publications of the Astronomical Society of the Pacific 129(976), 065001 (2017).

[8] Petit, C., Sauvage, J.-F., Fusco, T., Sevin, A., Suarez, M., Costille, A., Vigan, A., Soenke, C., Perret, D., Rochat, S., and et al., "Sphere extreme ao control scheme: final performance assessment and on sky validation of the first auto-tuned lqg based operational system," Adaptive Optics Systems IV (Aug 2014).

[9] Hardy, J. W., [Adaptive optics for astronomical telescopes], Oxford Univ. Press, New York [u.a.] (1998). 
[10] Conan, J.-M., Rousset, G., and Madec, P.-Y., "Wave-front temporal spectra in high-resolution imaging through turbulence," J. Opt. Soc. Am. A 12, 1559 (Jul 1995).

[11] Meimon, S., Petit, C., Fusco, T., and Kulcsar, C., "Tiptilt disturbance model identification for Kalmanbased control scheme: application to xao and elt systems," J. Opt. Soc. Am. A 27, A122 (Sep 2010).

[12] Agapito, G., Quirs-Pacheco, F., Tesi, P., Riccardi, A., and Esposito, S., "Observer-based control techniques for the lbt adaptive optics under telescope vibrations," European Journal of Control 17, 316326 (Jan 2011).

[13] Isermann, R. and Münchhof, M., "Identification of dynamic systems," (2011).

[14] Keck, A., "Accelerometer-based online reconstruction of vibrations in extremely large telescopes," Proceedings of the 19th IFAC World Congress (Aug 2014).

[15] Böhm, M., Glück, M., Keck, A., Pott, J.-U., and Sawodny, O., "Improving the performance of interferometric imaging through the use of disturbance feedforward," JOSA A 34(5), A10-A21 (2017).

[16] Glück, M., Pott, J.-U., and Sawodny, O., "Simulation of an accelerometer-based feedforward vibration suppression in an adaptive optics system for micado," in [SPIE Astronomical Telescopes+ Instrumentation], 99093N-99093N, International Society for Optics and Photonics (2016).

[17] Böhm, M., Pott, J.-U., Kürster, M., Sawodny, O., Defrère, D., and Hinz, P., "Delay compensation for real time disturbance estimation at extremely large telescopes," IEEE Transactions on Control Systems Technology 25(4), 1384-1393 (2017). 